\title{
Tangence
}

\section{Texte littéraire et référence}

\section{Philippe Hamon}

Numéro 44, juin 1994

La référence littéraire

URI : https://id.erudit.org/iderudit/025810ar

DOI : https://doi.org/10.7202/025810ar

Aller au sommaire du numéro

Éditeur(s)

Tangence

ISSN

0226-9554 (imprimé)

1710-0305 (numérique)

Découvrir la revue

Citer cet article

Hamon, P. (1994). Texte littéraire et référence. Tangence, (44), 7-18.

https://doi.org/10.7202/025810ar d'utilisation que vous pouvez consulter en ligne.

https://apropos.erudit.org/fr/usagers/politique-dutilisation/ 


\section{Texte littéraire et référence*}

\section{Philippe Hamon}

Histoire fortement encombrée, philosophiquement et linguistiquement, que celle du concept de référence. Paradoxalement la référence, l'acte de faire par des signes référence à quelque chose, est à la fois la chose du monde la mieux partagée (parler, c'est toujours parler de quelque chose, parler $\grave{a}$ quelqu'un, et c'est toujours poser une source réelle d'énonciation) et la plus difficile à décrire. Reprendre aujourd'hui la question, après une décennie d'approches immanentes du problème (l'analyse des problèmes de la co-référence et de l'auto-référence ayant pris le pas sur celle de la référence), n'est pas simple. Surtout après le passage quelque peu décapant des diverses sémiotiques et théories scientifiques contemporaines, voire des diverses psychanalyses ${ }^{1}$. Dans L'homme et la coquille, Valéry parlait déjà du "mollusque de référence "qu'est devenu selon lui, après Einstein, le monde que nous nous efforçons de penser ${ }^{2}$. Et si le retour du problème de la référence aujourd'hui, à une époque comme chacun sait "postmoderne", est général, les débats - souvent vifs entre d'une part les "immatérialistes" qui soulignent que notre rapport au réel est totalement médiatisé par des prothèses

* Le présent texte reprend, avec quelques ajustements et mises à jour, un article publié en mars 1987 dans le $\mathrm{n}^{\circ} 53$ ( La référence. Points de vue linguistique et logique $)$ des Travaux du Centre de recherches sémiologiques, Université de Neuchâtel, Suisse.

1 Pour de nombreux psychanalystes, le réel, le référent, c'est toujours l'absent du discours: : Démasquer le réel est le travail du psychanalyste. Le réel, c'est ce qui résiste, insiste, existe irréductiblement et se donne en se dérobant comme jouissance, angoisse, mort ou castration ", écrit S. Leclaire dans Démasquer le réel, Paris, Seuil, 1971, p. 11. D'un point de vue sémiotique et littéraire, voir les mises au point d'un numéro spécial de la revue Fabula: "Les référents du roman" (Lille, $\mathrm{n}^{\circ} 2$, octobre 1983).

2 * Einstein a dressé une pieuvre à capturer et à dévorer toute géométrie [...], mais [aussi] le temps, la matière et la pesanteur, et bien d'autres choses encore insoupçonnées des Grecs qui, broyées et digérées ensemble, font les délices du tout-puissant mollusque de référence" (Souligné par P. Valéry, Euvres, tome I, Paris, Gallimard, Bibliothèque de la Pléiade, 1968, p. 903). 
8

technologiques ${ }^{3}$, et d'autre part ceux qui veulent témoigner de "l'entêtement du référent à être toujours là " pour une réappropriation (sensorielle notamment) du réel ${ }^{5}$, ne contribuent pas toujours à éclaircir la notion.

Choisir un point de vue "littéraire" sur la question risque de ne rien arranger, du fait du statut particulièrement flou de l'objet même Littérature: objet réajustable au gré de l'Histoire et de la mutation des cultures, et que ni les linguistes ${ }^{6}$, ni les spécialistes de la communication, ni bien sûr les "littéraires" eux-mêmes, n'ont été capables de définir de façon satisfaisante. Quelques critères assez hétéroclites peuvent cependant être retenus, dont il est difficile pourtant de savoir s'ils sont nécessaires et suffisants pour poser une différence: 1) l'éventuelle question de la littérarité ne recouvre certainement pas celle de la fictionalité du texte; 2) le texte littéraire, comme le jeu, comme le sport, exacerbe plutôt qu'il ne neutralise notre attention au réel, si émoussée dans la vie courante et quotidienne par toute une série d'automatismes; 3) le texte littéraire est un texte écrit, différê, dissocié de son contexte d'énonciation du réel, donc un "carrefour d'absences" (l'auteur n'est pas là pour le lecteur, et réciproquement, et le contexte réel de l'un et de l'autre ne peut être qu'une reconstruction-reconstitution a priori (pour l'auteur) et a posteriori (pour le lecteur)) - la question à poser devenant donc la suivante: quel est le statut du référé dans le différé? 4) le texte littéraire entre dans une communication $a ̀$ postes flottants et interchangeables (l'auteur est à lui-même son propre et son premier lecteur; le lecteur est le véritable actualisateur du texte écrit, donc le véritable auteur de ce dernier; l'auteur est un succédané de son texte voir Montaigne: "Mon livre m'a fait" - comme le référent est un produit du texte, comme le texte construit son propre code; l'ênonciation en est peu localisable et toujours plus ou moins

3 Voir les essais de J. Baudrillard, Simulacres et simulations, Paris, Galilée, 1981, d'U. Eco : La guerre du faux (Paris, Grasset, 1985) et l'exposition * Les immatériaux " organisée au Centre Pompidou à Paris en 1985.

4 R. Barthes, La chambre claire, Paris, Gallimard/Seuil, 1980, p. 17.

5 Pour une réhabilitation du référent, voir $M$. Serres, Les cinq sens, Paris, Grasset, 1985, et F. Dagognet, Rematêrialiser, Paris, Vrin, 1985.

6 Voir par exemple É. Benveniste, "Sémiologie de la langue ", dans Problèmes de linguistique générale, Paris, Gallimard, 1974, tome II, et J. Searle, "The logical status of fictional discourse * (1975), traduit en français dans Sens et expression, Paris, Minuit, 1982. 
polyphonique $)^{7}$. La littérature définit donc une sorte d'aire de jeu pour des partenaires disjoints, mais un jeu non "gratuit" dans la mesure à la fois où il reformule règles et normes, où il les suit tout en les réélaborant, et où il informe et remodèle perpétuellement notre référence au réel ${ }^{8}$. Finalement, le littéraire aurait tendance à définir son objet non pas tant comme une essence, comme une pratique sémiotique dotée d'une quelconque et radicale spécificité, que comme un laboratoire permanent de refonte des systèmes axiologiques qui médiatisent notre rapport au réel, que comme un poste d'observation pour étudier les opérations de réécriture: réécritures inter-sémiotiques réécrivant les textes (ensembles de signes discrets) en fantasmes (ensembles d'images et de scénarios individuels psychiques) ou en idéologies (ensembles de consignes collectives et de valeurs institutionalisées), ou les idéologies en fantasmes, et réciproquement, chacun de ces trois ensembles, de surcroît, pouvant être défini comme "l'état provisoirement en équilibre d'un groupe de transformations" (C. Lévi-Strauss), transformations d'ordre interne, intra- ou intertextuelles (un texte est toujours la réécriture d'autres textes), intrafantasmatiques (un fantasme n'est souvent que la réécriture d'un autre fantasme) ou intra-idéologiques (une idéologie est un système homéostatique, un ensemble perpétuellement réajustable, "récupérateur", qui réécrit d'autres idéologies ou d'autres états antérieurs ou périmés de la même idéologie). Le "littéraire" serait donc l'analyste qu'intéresserait l'observation du groupe complexe de ces neuf opérations de transformation-réécriture:

7 Voir, dans la perspective des travaux de Bakhtine, l'essai d'O. Ducrot, Le dire et le dit, Paris, Minuit, 1984, où l'auteur critique "la théorie de l'unicité de l'énonciation" (p. 189).

8 La littérature fabrique et configure notre référence au même titre que bon nombre d'autres pratiques. Ne dit-on pas de telle situation réelle: *C'est du Kafka ", ou : "C'est courtelinesque *, ou de tel personnage réel : “C'est un personnage balzacien"? Voir, à cet égard, le mot souvent cité de Proust à Thibaudet à propos de Flaubert : *[Flaubert est] un homme qui, par l'usage entièrement nouveau et personnel qu'il a fait du passé défini, du passé indéfini, du participe présent, de certains pronoms et de certaines prépositions, a renouvelé presque autant notre vision des choses que Kant avec ses catégories, les théories de la connaissance et de la réalité du monde extérieur. * 


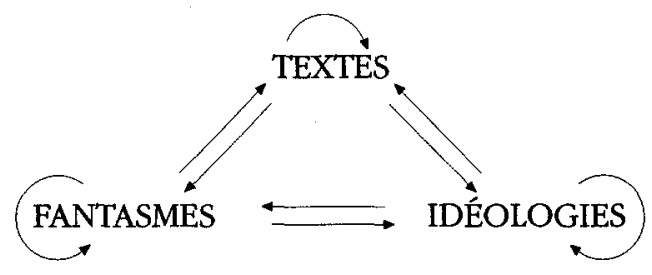

Ces opérations définissent non seulement les opérations fondamentales de "la littérature", mais aussi ce qui va peut-être en constituer le "sujet", le matériau privilégié, ce qu'elle va choisir prioritairement de décrire. En sélectionnant ce qui, dans le réel, se présente sous la forme de textes, de fantasmes ou d'idéologies, la littérature nous rappelle aussi que de tels objets en reformulation perpétuelle sont tout aussi "réels " qu'un arbre, qu'un objet fabriqué ou qu'un matériau naturel, que la référence ne vise donc pas seulement des "choses" concrètes, mais que le réel est un carrefour de relations en refonte permanente (les "mours" du romancier), de normes, de "valeurs " 9 , et pas seulement une collection de "choses" discrètes à étiqueter terme à terme par une nomenclature.

Outre les problèmes de définition de son objet, plusieurs questions cruciales et spécifiques se posent donc rapidement au littéraire, questions qu'un traitement trop strictement logique ou linguistique du problème de la référence laisse, justement, en suspens. La première est celle-ci: le référent est-il neutre? Théoriquement, pour un écrivain, tout est référentialisable, il n'y a pas de référents privilégiés, il peut sélectionner le réel et lui donner configuration comme bon lui semble. Or on observe que chaque genre littéraire se donne au contraire une série de contraintes de sélection parfois très strictes (contraintes de "sujet", de "méthode", de "style", de "ton", etc.) même dans la littérature la plus contemporaine où, semble-t-il, n'importe qui peut écrire n'importe quoi n'importe comment à propos de n'importe quoi. Bien plus, on peut se demander s'il n'y a pas une "sollicitation" de certains référents privilégiés à "entrer en littérature". Valéry, toujours dans L'bomme et la coquille, réfléchit sur certains objets (la coquille, le cristal, la fleur, les rythmes naturels) qui, de par quelque

9 Voir sur ce point, sur les modes et lieux d'inscription de l'idéologie dans le textuel, P. Hamon, Texte et idéologie, Paris, PUF, 1984. 
particularité de leur configuration, "se détachent du désordre ordinaire de l'ensemble des choses sensibles", "exposent [leur] capacitê de séduction sensible", "m'engagent dans un étonnement et une attention" ${ }^{10}$. Ces objets-questions (naturels ou humains, donnés ou construits, configurés ou configurants?), objets complexes, "matière à doute " 11 , peuvent donc suggérer à un écrivain de vouloir en déconstruire les ambiguités, sont peut-être plus intéressants que d'autres à traiter, peuvent peut-être, plus que d'autres, suggérer leur intégration comme matériau à des récits, à des fantasmes ou à des idéologies. Même chose pour ces "zoèmes" dont parle C. Lévi-Strauss dans ses Mytbologiques, animaux réels qui, par leur configuration anatomique même (par exemple une raie ou un papillon, animaux à la fois très minces - vus par la tranche - et très larges - vus d'en dessus), par une sorte d'ambiguïté "naturelle" donc, sont particulièrement propres à entrer comme acteurs dans le système des combinatoires, des interrogations et des questionnements mythiques. Et pourquoi pas, sur le symétrique de ce concept de "zoème" avancé par C. LéviStrauss, ne pas suggérer celui de "technème" (par exemple portes, fenêtres, murs, etc., objets architecturaux à la fois conjoncteurs et disjoncteurs, donc susceptibles d'illustrer toute problématique d'ambiguïsation ou de désambiguïsation) ${ }^{12}$ ? La question ne serait donc plus: comment l'écriture cite-t-elle le réel? mais plutôt: comment le réel sollicite-t-il l'écriture?

Une deuxième question proprement "littéraire" concernerait l'effet de certains référents, dons de leur référence sur, plutôt que leur référence $a \grave{a}$. Si le référent n'est pas forcément un matériau "neutre", l'acte même de référer n'est sans doute pas non plus, pour le lecteur comme pour son producteur, un acte "neutre". Aristote, bien avant Pascal dont la formule voisine est plus

10 P. Valéry, op. cit., p. 887 et 889. Le texte ne "cite " pas la coquille, c'est la coquille qui est “sollicitation - (p. 886) et est un "excitant * (p. 886, 906).

11 P. Valéry, op. cit., tome II, dans Eupalinos ou l'architecte (c'est un mot de Socrate à Phèdre) où Valéry reprend une scène semblable de "trouvaille" d'un objet "excitant " sur le bord de la mer, objet qui suscite une réflexion "entre le construire et le connaître (p. 115). Valéry réécrit là, sans le dire, le premier chapitre des Entretiens d'Ariste et d'Eugène du père Bouhours (1671) et ses réflexions sur le rythme comme principe configurationel du sensible.

12 Sur les "zoèmes", voir C. Levi-Strauss, Mythologiques, Paris, Plon, 1971, tome IV, p. $68,482,500$, etc. Pour le concept de a technème *, voir P. Hamon, Expositions, littérature et architecture, Paris, Corti, 1989. 
connue ${ }^{13}$, se demandait déjà de quelle nature était ce "bénéfice", cette "prime de plaisir", dira Freud, qui peut s'attacher, indépendamment du référent, à l'acte même de référer. Toute la réflexion classique sur la Mimesis tourne autour de ce problème, de la Poétique d'Aristote à "l'inquiétante étrangeté " de Freud, problème où s'articule inextricablement le problème du savoir, celui de la connaissance, celui de la reconnaissance et celui du plaisir. De fait, à y regarder de près, la référence a toujours été l'objet de réglementations sourcilleuses de la part des diverses théories littéraires, théologies, ou rhétoriques, qui l'ont prise en charge: problème de la censure et des tabous de la représentation, querelles iconoclastes diverses, débats autour de la description (lieu textuel que l'on "saute"), révolutions littéraires qui se sont toutes faites au nom d'un retour "réaliste" aux "choses", dispute autour du statut de la catachrèse, prescriptions ou proscriptions de la "figure", cette utilisation du langage qui, à la fois, "porte absence et présence, plaisir et déplaisir " ${ }^{14}$. Si le problème central de la référence devient celui de la reconnaissance (face à celui, peut-être plus proprement linguistique, de la comprébension des énoncés: reconnaître un signe, reconnaître une chose, et reconnaître la relation d'un signe à une chose), c'est toute une typologie des plaisirs et des déplaisirs qui doit être élaborée, axée sur la reconnaissance et le savoir : plaisir à reconnaître quelque chose en sachant ce que c'est, angoisse à ne pas se reconnaître (sur une de ses photographies), ou à reconnaître quelque chose (ou quelqu'un) ne ne sachant pas ce que (qui) c'est, etc. Il est possible, d'ailleurs, que la référence se fasse toujours plus ou moins sur le mode de la dénégation, de la prétérition, de l'euphémisme ${ }^{15}$, ou de la réécriture contradictoire: crainte, notamment de dire ce dont

13 On peut effectivement mettre en parallèle Aristote: "Nous avons plaisir à regarder les images les plus soignées des choses dont la vue nous est pénible dans la réalité, par exemple les formes d'animaux parfaitement ignobles ou de cadavres * (Pótique, éd. Dupont-Roc-Lallot, Paris, Seuil, 1980, p. 43), et Pascal: "Quelle vanité que la peinture qui attire l'admiration par la ressemblance des choses dont on n'admire point les originaux. (Pensées, éd. Brunschvicg, Hachette, s.d., p. 389).

14 Pascal, op. cit., p. 636. Innombrables sont en effet les figures de l'ancienne rhétorique qui s'efforcent de codifier minutieusement l'absence de référence (l'euphémisme, la litote, la réticence, l'ironie, la périphrase, etc.).

15 Sur l'euphémisme, voir É. Benveniste, Problèmes de linguistique générale (tome I, Paris, Gallimard, 1966, p. 308 et s.). Valéry à nouveau: "Ầ mesure que l'on approche du réel, on perd la parole . (Euvres, op. cit., tome II, p. 554). 
nous sommes certains, ce que nous ne pouvons pas ne pas connaître, la seule chose à laquelle on ne puisse pas ne pas croire, notre mort. Pourquoi, en particulier, ce plaisir "réaliste" qui est celui de tous les lecteurs de romans, qui consiste à lire des "êtres de papier", des êtres fictifs (Julien Sorel, Madame Bovary, etc.) comme s'ils étaient réels, sinon pour éviter de penser l'inverse insupportable, que des êtres réels (nous-mêmes) peuvent un jour devenir - ce qui va certainement nous arriver quand nous serons morts - des êtres de papier, des êtres de fiction, des êtres de récit (qui n'existeront que dans et à travers les récits et les souvenirs de ceux qui nous survivront)? Et Freud, esquissant dans le thème des trois coffrets ${ }^{16}$ ce que l'on pourrait considérer comme les linéaments d'une grammaire de la réécriture fantasmetexte, montre bien que ce thème littéraire qu'il décrit ("Un homme choisit librement la chose la plus positive entre plusieurs choses") est la réécriture inversée d'une certitude informulable touchant le référent absolu, la mort ("la mort - ce qu'il y a de plus négatif au monde — prend inéluctablement l'homme"). Le "détail" qui arrête donc le lecteur dans sa lecture, ce point poignant de l'œuvre qui fascine par son poids et sa charge "réaliste" de "déjà vu", par sa "précision" ou par la "minutie" de son intitulé, cette "émeute du détail" (Baudelaire) qui vient "couper le fil" du récit, qui "tranche" par son insignifiance même sur le continuum du texte, ne serait pas tant simple "défaut " ou "jeu " dans l'arrangement textuel, simple intrusion d'un élément "extérieur" à l'œuvre (le réel brut), ou simple "introduction d'un élément inattendu dans une structure "(définition classique du fait de style en général), mais serait plutôt ce qui me renverrait impérativement à la pensée de cette seule réalité dont je sois sûr et certain, le fait que je vais mourir un jour ${ }^{17}$. Le réel dans l'œuvre ne viendrait donc pas tant d'un mimétisme avec ce qui lui est extérieur, de la réintroduction d'un déjà-vu, mais d'un "écrasement" des conditions mêmes de toute prise en charge et énonciation du réel (le temps, l'espace; la distinction passé / présent/futur, ici / ailleurs): le réel du texte et la réalité de la littérature, ce serait alors "voir

16 Dans Essais de psychanalyse appliquée [trad. fr.], Paris, Gallimard.

17 Nous renvoyons bien sûr ici au Barthes de La chambre claire (op. cit.), où la notion de "punctum " prolonge et enrichit celle de "détail " développée en 1968 dans l'article "L'effet de réel" (Communications, $\mathrm{n}^{\circ} 11$ ). Sur ces points, voir également $\mathrm{P}$. Hamon: "Thème, thématique et effet de réel ", Poétique, $n^{\circ} 64,1985$. 
les souvenirs futurs des gens" (Nabokov) ${ }^{18}$, ou "voir le passé regarder la mort au futur" (Barthes) ${ }^{19}$.

Un point de vue "littéraire" sur le problème de la référence serait donc un point de vue qui tiendrait compte systématiquement de ces stratégies et de ces spécificitês retorses de l'objet décrit et différé, et du statut non moins retors du "réel " tel qu'il est construit-reconstruit dans et par ces opérations de reformulation.

Ceci posé, il conviendrait, bien sûr, de prolonger la réflexion sur la question. Laquelle question ne pouvant plus être: "Comment la littérature fait-elle référence au réel?" mais devenant plutôt: "Comment la littérature nous fait-elle croire qu'elle se réfère au réel?" J'ai, ailleurs, essayé d'engager cette réflexion ${ }^{20}$, sous l'égide d'une phrase extraite de Bouvard et Pécuchet ${ }^{21}$, en faisant de l'acte (littéraire) de référer essentiellement une manipulation (sans donner à ce terme aucune acception péjorative), un "faire-croire", donc un cas particulier de discours persuasif (au même titre que d'autres types de discours persuasifs voisins: le discours de séduction, le discours d'intimidation, le discours de tentation, le discours pédagogique, etc., tous discours du fairesavoir ou du faire-vouloir qui tous essaient de faire faire quelque chose à quelqu'un), discours dont on pourrait ainsi paraphraser le projet: faire croire que le référé existe (a existé, existera, ou pourrait exister) et que des garanties peuvent être données au

18 Voir, de Nabokov, le très beau texte intitule "Guide de Berlin ", texte de 1925 publié en 1930 en russe (traduction française dans Détails d'un coucher de soleil, Paris, Julliard, 1985).

19 Voir La cbambre claire, op. cit., p. 144-151. L'expression de "temps écrasé "se trouve à la page 151. R. Barthes a emprunté cette notion de "punctum" aux réflexions de W. Benjamin sur la photographie. Ce dernier parle du spectateur de la photo amalgré lui forcé de chercher dans une pareille image la petite étincelle de hasard, d'ici et de maintenant, grâce à laquelle le réel a, pour ainsi dire, brûlé le caractère d'image [...] lieu imperceptible où, dans la façon d'être singulière de cette minute depuis longtemps révolue, niche encore aujourd'hui l'avenir " (Essais, I, [trad. fr.], Paris, Médiations, 1982, p. 153).

20 Dans "Note sur la référence ", Fabula, op. cit., et dans "Thème, thématique et effet de réel ", Poétique, op. cit.

21 "Sans connaître les modèles, ils [Bouvard et Pécuchet] trouvaient ces peintures [les descriptions des romans de Walter Scott] ressemblantes, et l'illusion était complète.. Maupassant également, dans sa préface à Pierre et Jean (1887), insistera sur ce point: *Faire vrai consiste [...] à donner l'illusion complète du vrai $[\ldots]$ Les réalistes de talent devraient plutôt s'appeler des Illusionnistes." 
lecteur quant au sujet de l'énonciation d'une part, quant au sujet de l'énoncé d'autre part.

Bien sûr, reprendre le problème de la référence dans les termes et dans la problématique de celui de la croyance (qu'est-ce que croire?) risque de ne pas simplifier les choses. Ce problème, remarquons-le en passant, est au centre de la recherche la plus contemporaine en sciences humaines, recherche qui, centrée sur les trois objets sémiotico-symboliques que nous avons retenus plus haut (le texte, l'idéologie, le fantasme), rencontre inéluctablement ce problème de la croyance, soit comme objet (le sociologue et l'historien, par exemple, se demandent pourquoi et comment telle civilisation ou telle époque croit en/à telle ou telle chose; le psychanalyste, quel est le statut, dans l'analyse, de la "reconnaissance", etc.), soit comme question épistémologique fondamentale (par exemple, l'utilisation de schèmes narratifs par l'historien n'est-elle pas destinée à fabriquer des effets de créance, un effet de vraisemblable, chez le lecteur? En quoi les nécessités pédagogiques d'avoir à rendre compte d'une analyse à une communauté scientifique conditionnent-elles implicitement la description elle-même, etc.) ${ }^{22}$. Il conviendrait sans doute, tout d'abord, d'analyser avec plus de précision ce qu'il y a de proprement "contractuel " dans l'acte de référence: tout contrat suppose programme, système de sanctions et de récompenses, promesse et engagement à respecter des règles, garanties, et tout cela doit avoir ses équivalents sémiotiques dans l'acte de référence. Ce dernier est donc une sorte de "contrat fiduciaire", de "pacte de

22 Sur le champ sémantique du verbe croire, voir É. Benveniste, Le vocabulaire des institutions indo-européennes, Paris, Minuit, 1970, tome I, "Créance et croyance", p. 171 et s.). Voir également les travaux de Michel de Certeau, L'écriture de l'Histoire, Paris, Gallimard, 1975, et "Manières de croire * dans L'invention du quotidien, I, 10/18, 1980; de M. Detienne, Les mâ̂tres de vérité dans la Grèce arcbaïque, Paris, Maspero, 1967; de P. Veyne, Les Grecs ont-ils cru à leurs mythes?, Paris, Seuil, [1983] 1992; de R. Boudon, L'idéologie ou l'origine des idées reçues, Paris, Fayard, 1986; et, sur un plan sémiotique, ceux d'A. J. Greimas, "Le savoir et le croire", dans Du sens, II, Paris, Seuil, 1983. Sur les liens entre croire et savoir, sur leur association ou dissociation réciproque (voir les techniques du quiproquo au théâtre; voir la formule: "Je sais bien, mais quand même...v), on peut retourner à Valéry: "Notre savoir consiste en grande partie à "croire savoir", et à croire que d'autres savent (L'bomme et la coquille, op. cit.). Parlant de la mimesis dans sa Poétique (VI, 48b), Aristote parle aussi du plaisir de savoir, lié au plaisir de reconnaître. 
16

référence" où s'intriquent croyance et créance, et qui fait donc de la croyance un acte qui ne saurait être purement passif, mais qui est au contraire une activité de vérification et d'interprétation continue coincidant chez le lecteur avec l'acte de la lecture. Ce pacte référentiel serait sous-jacent à tout texte différé, quel que soit son genre (que ce dernier se présente comme "réaliste", "merveilleux", "lyrique", "fantastique ", etc.), et indépendamment de toute référence au couple vrai-faux, ou fiction-réalité, catégories qui sont d'un très faible rendement pour étudier textes, idéologies ou fantasmes. L'analyse devra donc voir de quelle manière chaque texte écrit (différé) construit son propre programme de croyance à ce qu'il dit, comment il défère son référent à comparaître devant le tribunal du lecteur: voir en particulier comment s'articule le croire à (je crois à ce qui est dit par l'énoncé, à l'existence passée, présente, future ou simplement possible de ses référents, à la conformité de la description avec le décrit, et j'évalue les garanties qui me sont données quant à cette existence et à cette conformité), le croire en (j'ai moi lecteur, besoin de la médiation d'une certaine image du donateur de l'énoncé, de l'image d'un narrateur-auteur autorisé, $\mathfrak{j}$ 'ai besoin de croire en lui, en la compétence ou en la franchise de son dire, à son "expérience", etc., donc de garanties quant au(x) sujet(s) de l'énonciation), et le croire avec (l'effet de réel est inséparable des institutions, des productions collectives et des communautés - idéologies, académies, encyclopédies, avant-gardes, etc. - qui le prennent en charge et le garantissent). Le croire $\dot{a}$, probablement, passera toujours par la médiation d'un croire en et d'un croire avec, la référence $\grave{a}$ par une déférence en et une conférence $a v e c^{23}$; en d'autres termes, la référence (textuelle) passe par l'image (intratextuelle) de son énonciation et par celle (intertextuelle) d'une communauté. Bien sûr, plusieurs stratégies de persuasion sont possibles, et à plusieurs niveaux d'organisation du texte. La manipulation fiduciaire d'un lecteur passera sans doute d'abord par la mise en scène immédiate, dans le texte même et quel que soit le "sujet", de divers scénarios plus ou moins figuratifs particuliers, par le montage d'une sorte de "dramaturgie épistémique " incorporée destinée à mettre en scène et à représenter les objets, les actions et les acteurs de l'acte même de croire: héros engagés

23 Voir l'expression "Texte de référence", qui désigne souvent la "littérature * que l'on doit obligatoirement citer sur tel ou tel sujet. Le cliché est souvent le signal, dans un texte, d'une "communauté de croyance". 
dans des quêtes de vérité; exposition, circulation et évaluation de stigmates, de signes, de simulacres, de reliques (ce à quoi l'on croit) ${ }^{24}$ dans un récit; paraboles plus ou moins symboliques mettant en scène des "opposants", des "traîtres" à ces quêtes de vérité (convaincre quelqu'un c'est toujours plus ou moins le "vaincre"; il y a toujours une dimension polémique dans toute manipulation); personnages qualifiés ou disqualifiés dans leur compétence à distinguer le réel du fictif, le vrai du faux; détectives, herméneutes ou évaluateurs qualifiés ou disqualifiés dans leur aptitude à lire et à interpréter les symptômes et les signaux du monde; personnages d'ironisants ou d'hypocrites, de naifs ou de crédules; personnages engagés dans des aventures juridiques, sentimentales et financières (donc impliquant créances, créanciers et crédits divers) toutes régies par des contrats, une normativité et des règles fortes, etc. ${ }^{25}$ Ainsi le lecteur, prenant connaissance de cette dramaturgie épistémique explicite (qui n'est donc qu'une "thématique" parmi d'autres proposées par le texte) pourra, y trouvant les analogues, les métaphores, les modèles ou les repoussoirs ridiculisés de ses propres stratégies herméneutiques, adhérant plus ou moins à la compétence ou à l'incompétence des personnages mis en scène, au succès ou à l'insuccès de leurs quêtes de vérité (Mademoiselle Cormon, l'héroïne de La vieille fille de Balzac, trop crédule, mourra vierge pour n'avoir pas su interpréter correctement les signaux du monde physique et du

24 La relique du "croyant * est bien souvent une partie d'un objet ou d'un corps, un "relief" ou une "trace ". Rappelons que, selon Jakobson et Barthes, le texte réaliste serait particulièrement "friand" de "détails", de métonymies et de synecdoques, et par conséquent le fragmentaire semblerait donc avoir quelque rapport privilégié avec l'effet de crédibilité : le précis est réputé vrai.

25 Ces scénarios, qui mettent en scène des personnages identifiables et distincts, sont des scénarios figuratifs. Il resterait à explorer les stratégies persuasives de la crédibilité qui passent par des procédés "abstraits", non figuratifs, "effets de structure " jouant le rôle de tenant-lieu de l'auteur absent: quel est, par exemple, et indépendamment de ses référents, cet a effet de congruence croissante " (M. Riffaterre) que peut provoquer l'expansion d'une "liste" ou le dépliement d'un système descriptif? Quel type de collaboration existe-t-il entre les structures narratives d'un énoncé et des structures argumentatives-logiques comme le syllogisme ou l'enthymème (rôle du post boc ergo propter boc)? Quels sont exactement les "effets d'autorité" provoqués par une citation? Toute relation structurale perçue dans un énoncé ne donne-t-elle pas implicitement une "image de narrateur" fiable, maîtrisant son énoncé, "cohérent", donc crédible? Quels sont les "effets probatoires" des diverses structures textuelles? 
monde social), développer lui-même les conditions de sa propre croyance à ce que dit le texte. L'inventaire et la typologie des composants de cette scène épistémique thématisée seront sans doute assez aisés. Il sera sans doute, déjà, un peu moins aisé de dresser la typologie des "images de l'énonciation qui sont véhiculées à travers l'énoncé "26, images d'actants énonciatifs déductibles de l'énoncé, plus ou moins implicites ou explicites (de "l'historien" balzacien aux intrusions fracassantes, au "ton" verlainien, en passant par le "naturel "stendhalien, ou par "l'objectivité" zolienne, et par toutes les manières et maniérismes d'écriture, toutes images en creux d'une énonciation différée), images reconstruites sporadiquement et localement par la lecture, groupe d'images suggérant des narrateurs plus ou moins crédibles, qui vont venir constituer peu à peu comme une deuxième scène épistémique, scène plus ou moins monologique ou polyphonique par la médiation et le détour de laquelle (le croire en et le croire avec) passera obligatoirement l'effet de croyance final et global (le croire a). Mais l'interprétation de la relation (homologie, dissociation, implication, contradiction, etc.) entre la (les) scène(s) de créances explicitement montée(s) et énoncé(s) dans le texte, et la (les) scènes énonciatives implicite(s) et déduite(s) $d u$ texte ne va pas de $\operatorname{soi}^{27}$, ainsi que leur interprétation et que l'attribution ultime de leur combinaison à quelque créancier unique, localisable et originel (un "auteur", par exemple). La littérature, communication médiatisée, différée, dont les composants sont dissociables et non localisables, vit dans et de la nostalgie du performatif, cet acte de langage où parler, agir, remplir un contrat, référer, donner des garanties et produire le référent sont indissociables, sont immédiats, et associés à une source réelle identifiable.

26 O. Ducrot, Le dire et le dit, op. cit., p. 174. (Je souligne: à travers et non pas dans.) La question de la posture d'énonciation ironique (vs posture d'énonciation "sérieuse") pose le problème de la "distance" de (l'image de) l'énonciateur vis-à-vis du référent de son énonciation. Elle est souvent la manifestation d'une triple distance: 1) distance de l'énonciateur vis-à-vis de son énonciation; 2) distance d'une partie de l'énoncé vis-à-vis d'une autre partie de ce même énoncé; 3) distance de l'énoncé vis-à-vis d'un autre énoncé (parodie, pastiche, réécriture, écho, mention).

27 Voir, par exemple, la distorsion qui peut exister entre le croire et le savoir des personnages et le croire et le savoir du narrateur dans tous les textes "à suspense - ou "fantastiques ; ou, dans le genre pictural de la nature morte de type "Vanitas" du Xvil ${ }^{\mathrm{e}}$ siècle, entre ce qui est peint (la mise en scène de la vanité de la référence au réel par les cinq sens) et la manière de peindre (très " réaliste", "illusionniste ", voire en "trompe-l'œil "). 